Pathologe 2014 · [Suppl 2] · 35:293-293

DOI 10.1007/s00292-014-1980-5

Online publiziert: 19. September 2014

(c) Springer-Verlag Berlin Heidelberg 2014

A. M. Müller

Zentrum für Kinderpathologie und Pathologie, MVZ Venusberg, Universitätsklinikum Bonn

\title{
Bericht der AG Kinder- und Fetalpathologie 2014
}

Trophoblastinvasion bei Präeklampsie einschließlich einer dadurch bedingten plazentaren Protektion vor Hypertonie und einen Kompensationsmechanismus bei durch Rauchen gestörtem Gleichgewicht zwischen Trophoblastproliferation und Zottendifferenzierung.

Pinkus et al. (Graz/Kiel) zeigten in ihrem Beitrag zu Regulation des TGFbeta-signaling pathway in Nephroblastomen die deregulierende Wirkung von verschiedenen miRNA auf den „TGF-beta-signaling pathway“. Die Arbeitsgruppe Stiegelbauer/Gürtl (Graz/Kiel) wies anhand transfezierter HEK293-Zellen in Nacktmäusen eine Überexpression von WBSCR („Williams-Beuren syndrome critical region“) 17 nach und fragten konsequenterweise, ob die Überexpression nicht einen Beitrag zur Entwicklung maligner Tumoren trägt. Obduktionsbefunde bei abortierten Feten mit Trisomie 18 im Obduktionsgut der Universitätspathologie Leipzig in den Jahren 2006-2013 demonstrierten Bauer/Horn (Leipzig), über eine Studie zur Bedeutung der fetalmorphologischen Obduktionsbefunde bei mittels FISH bestätigter Mosaiktetrasomie 12p berichteten Schoner et al. (Marburg). Rügger et al. (Zürich) zeigten erste Befunde der minimal-invasiven, bildgebungsgeführten virtuellen Autopsie im Vergleich zur konventionellen Autopsie bei Feten, Neonaten und Kleinkindern.

Über die schwierige Diagnostik von PTLD („post-transplant lymphoproliferative disorders") nach Lebertransplantation berichteten Sändig et al. (Leipzig/ Halle), über den weltweit 10. Fall eines kongenitalen unreifen Teratoms des $\mathrm{Na}$ sopharynx mit Ausdehnung bis nach intrakraniell Opitz et al. (Leipzig), über ei- ne Zwillingsschwangerschaft einer männlichen frühen Blasenmole und eines weiblichen Feten Scheil-Bertram et al. (Wiesbaden), über ein Pylorusdrüsenadenom Märkl et al. (Augsburg).

Ein Highlight der Sitzung stellten die Keynote Lectures dar. Hier sprach Prof. Cord Langner (Graz) über die aktuellen diagnostischen Leitlinien für die Diagnostik chronisch-entzündlicher Darmerkrankungen (CED) bei Kindern unter besonderer Berücksichtigung der diagnostischen Unterschiede zur adulten CED. Prof. Alex Knisley (London) diskutierte anhand des Themas „Neues in der Cholestasediagnostik " die enge, einander befruchtende Interaktion der histo- und molekularpathologischen Cholestasediagnostik.

\section{Korrespondenzadresse}

Prof. Dr. A. M. Müller

Zentrum für Kinderpathologie und Pathologie, MVZ Venusberg, Universitätsklinikum Bonn Sigmund-Freud-Str. 25, 53127 Bonn annette.mueller@ukb.uni-bonn.de

\section{Einhaltung ethischer Richtlinien}

Interessenkonflikt. A. M. Müller gibt an, dass kein Interessenkonflikt besteht.

Dieser Beitrag beinhaltet keine Studien an Menschen oder Tieren.

The supplement this article is part of is not sponsored by the industry. 